\title{
二個の共線等長き裂を有する圧電厚板の電気熱弾性応答*
}

$$
\text { 上田整*1, 谷吉 郎*2 }
$$

\section{Thermal Stress Intensity Factors for Two Coplanar Cracks in a Piezoelectric Strip}

\author{
Sei UEDA*3 and Yoshiro TANI
}

${ }^{* 3}$ Department of Mechanical Engineering, Osaka Institute of Technology,
5-16-1 Omiva, Asahi-ku, Osaka-shi, Osaka, 535-8585 Japan

This work is concerned with the thermoelectromechanical fracture behavior of two coplanar cracks in a piezoelectric material strip under a uniform heat flow far away from the crack region. The crack faces are supposed to be insulated thermally and electrically. By using the Fourier transform, the thermal and electromechanical problems are reduced to singular integral equations, respectively, which are solved numerically. Numerical calculations are carried out, and detailed results are presented to illustrate the influence of the crack location and length on the thermal stress intensity factors.

Key Words : Piezoelectric Material Strip, Elasticity, Fracture Mechanics, Thermal Stress, Stress Intensity Factor, Integral Transform

\section{1. 緒言}

圧電材料はセンサ, アクチュエータなどとして用い られ，現代の科学技術を支えている重要な材料となっ ており，その電気破壊力学に関する研究が進められて いる. また，これらの圧電材料と構造材料を組み合わ せることで, 熱的環境下における複合材料の変形制御 を可能とする知的圧電複合材料システムを実現できる (1).そのため, 熱的環境下にある均質圧電材料中の二 次元き裂問題 (2) (6) や円板状き裂問題 (7) および傾斜 機能圧電材料 (FGPM) 中の二次元き裂問題 (8) (12) や 円板状き裂問題 (13),(14) などの研究結果が報告されて いる。また，著者らは，圧電材料の自由表面に垂直に 存在するき裂を対象とした電気熱弾性破壊力学解析を 行っている(15),(16)

一方, 材料内には多数のき裂が存在するため, 破壊 挙動に及ぼすき裂間相互干涉の影響を解明することも 重要である. Chen and Worswick らは，電気的機械 的負荷が作用する均質圧電材料中の共線等長き裂問題 （17）を解析しているが, 熱的負荷が作用する場合のき 裂間相互干涉を取り扱った研究例は見当たらないよう である。

* 原稿受付 2007 年 8 月 3 日。

*1 正員, 大阪工業大学工学部 (画535-8585 大阪市旭区大宮 516-1).

*2 学生員, 大阪工業大学大学院工学研究科.

E-mail : ueda@ med.oit.ac.jp
そこで本報では, 一様熱流が作用する圧電厚板を考 え，二個の共線等長き裂が自由表面に対して垂直に存 在する場合のき裂先端の熱応力拡大係数を理論解析し た．き裂面における電気的境界条件には, 工学上有効と される非透過型の条件を用いた (18). 解析には, Fourier 変換法を用い, 問題の解を特異積分方程式の解に導い て解く方法を採用した(19),(20). また, Gauss-Jacobi の 数值積分公式 (21) を用いて特異積分方程式を数值解析 し，温度分布およびき裂先端の熱応力拡大係数に及ぼ すき裂位置およびき裂長さの影響を明らかにした。

\section{2. 問題の設定と基礎式}

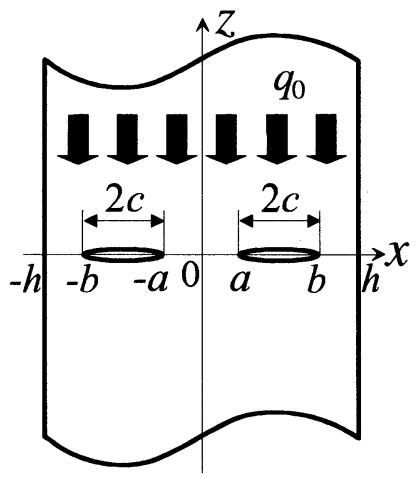

Fig. 1 Two coplanar cracks in a piezoelectric material strip. 
図 1 に示す直角座標系 $(x, y, z)$ において, $-h \leq x \leq$ $h$ の領域を占める圧電厚板を考え, $z=0$ 面上に長さ $2 c=b-a(0<a<b<h)$ の等長二き裂が $z$ 軸に関 して対称に存在するものとし, 平面ひずみを仮定する. また, 圧電厚板の分極方向を $z$ 軸方向とし, $z$ 軸の負の 向きに一様熱流 $q_{0}$ が作用するものとする. 弾性定数 を $c_{11}, c_{12}, c_{13}, c_{23}, c_{33}, c_{44}$, 圧電定数を $e_{15}, e_{31}, e_{33}$, 誘電定数を $\varepsilon_{11}, \varepsilon_{33}$, 温度応力係数を $\lambda_{11}, \lambda_{33}$, 熱伝導 率を $\kappa_{x}, \kappa_{z}$, 焦電定数を $p_{z}$ で表し, 下付添字 $x, y, z$ で座標軸方向を示すものとする。

材料の温度を $T(x, z)$, 静電ポテンシャルを $\phi(x, z)$, 変位ベクトルの成分を $u_{x}(x, z), u_{z}(x, z)$ とすると, 応 カテンソルの成分 $\sigma_{x x}(x, z), \sigma_{z z}(x, z), \sigma_{z x}(x, z)$ およ び電束密度ベクトルの成分 $D_{x}(x, z), D_{z}(x, z)$ は次の ように与えられる。

$$
\left.\begin{array}{rl}
\sigma_{x x} & =c_{11} \frac{\partial u_{x}}{\partial x}+c_{13} \frac{\partial u_{z}}{\partial z}+e_{31} \frac{\partial \phi}{\partial z}-\lambda_{11} T \\
\sigma_{z z} & =c_{13} \frac{\partial u_{x}}{\partial x}+c_{33} \frac{\partial u_{z}}{\partial z}+e_{33} \frac{\partial \phi}{\partial z}-\lambda_{33} T \\
\sigma_{z x} & =c_{44}\left(\frac{\partial u_{x}}{\partial z}+\frac{\partial u_{z}}{\partial x}\right)+e_{15} \frac{\partial \phi}{\partial x} \\
D_{x} & =e_{15}\left(\frac{\partial u_{x}}{\partial z}+\frac{\partial u_{z}}{\partial x}\right)-\varepsilon_{11} \frac{\partial \phi}{\partial x} \\
D_{z} & =e_{31} \frac{\partial u_{x}}{\partial x}+e_{33} \frac{\partial u_{z}}{\partial z}-\varepsilon_{33} \frac{\partial \phi}{\partial z}+p_{z} T
\end{array}\right\}
$$

直交異方性を対象とした熱伝導方程式は次式で表さ れる。

$$
\kappa^{2} \frac{\partial^{2} T}{\partial x^{2}}+\frac{\partial^{2} T}{\partial z^{2}}=0
$$

ここに, $\kappa^{2}=\kappa_{x} / \kappa_{z}$ である. また, 変位および静電ポ テンシャルに関する場の支配方程式は

$$
\begin{aligned}
& c_{11} \frac{\partial^{2} u_{x}}{\partial x^{2}}+c_{44} \frac{\partial^{2} u_{x}}{\partial z^{2}}+\left(c_{13}+c_{44}\right) \frac{\partial^{2} u_{z}}{\partial x \partial z} \\
& +\left(e_{31}+e_{15}\right) \frac{\partial^{2} \phi}{\partial x \partial z}=\lambda_{11} \frac{\partial T}{\partial x} \\
& c_{44} \frac{\partial^{2} u_{z}}{\partial x^{2}}+c_{33} \frac{\partial^{2} u_{z}}{\partial z^{2}}+\left(c_{13}+c_{44}\right) \frac{\partial^{2} u_{x}}{\partial x \partial z} \\
& +e_{15} \frac{\partial^{2} \phi}{\partial x^{2}}+e_{33} \frac{\partial^{2} \phi}{\partial z^{2}}=\lambda_{33} \frac{\partial T}{\partial z} \\
& e_{15} \frac{\partial^{2} u_{z}}{\partial x^{2}}+e_{33} \frac{\partial^{2} u_{z}}{\partial z^{2}}+\left(e_{15}+e_{31}\right) \frac{\partial^{2} u_{x}}{\partial x \partial z} \\
& -\varepsilon_{11} \frac{\partial^{2} \phi}{\partial x^{2}}-\varepsilon_{33} \frac{\partial^{2} \phi}{\partial z^{2}}=-p_{z} \frac{\partial T}{\partial z}
\end{aligned}
$$

問題の対称性を考慮して, $0 \leq x \leq h, 0 \leq z<\infty$ の 領域で解析することにすると, 温度場・電気熱弾性場 に関する境界条件式は次式となる.

$$
\left.\begin{array}{cr}
\partial T(x, 0) / \partial z=0 & (a<x<b) \\
T(x, 0)=0 & (0 \leq x \leq a, b \leq x \leq h)
\end{array}\right\}
$$

\section{3. 解 析}

温度場に関する支配方程式 (3) および境界条件式 (5), (6) は前報 (16) と同様であるため, 結果のみを示すと, 温度場 $T(x, z)$ は

$$
T(x, z)=T^{(1)}(z)+T^{(2)}(x, z)
$$

ここに, $T^{(1)}(z)$ は非擾乱温度場, $T^{(2)}(x, z)$ は擾乱温 度場を示し, それぞれ次式で与えられる。

$$
\begin{aligned}
& T^{(1)}(z)= \frac{q_{0}}{\kappa_{z}} z \\
& T^{(2)}(x, z)= \frac{1}{2 \pi i} \int_{-\infty}^{\infty} \frac{1}{s} L_{0}(s) \exp (-|s| \kappa z) \exp (-i s x) d s \\
&-\frac{2}{\pi} \int_{0}^{\infty} \frac{1}{s}\left[L_{1}(s) \exp (-s x / \kappa)\right. \\
&\left.\quad-L_{2}(s)\{\exp (-s x / \kappa)+\exp (s x / \kappa)\}\right] \\
& \quad \times \sin (s z) d s
\end{aligned}
$$

上式中の $L_{j}(s)(j=0,1,2)$ は前報 (16) と同様である. 特異電気熱弾性場は擾乱温度場 $T^{(2)}(x, z)$ のみに起 因することを考慮し, 変位べクトルの成分 $u_{x}(x, z)$, $u_{z}(x, z)$ および静電ポテンシャル $\phi(x, z)$ を次式で表す.

$$
\left.\begin{array}{l}
u_{x}(x, z)=u_{x}^{(1)}(x, z)+u_{x}^{(2)}(x, z) \\
u_{z}(x, z)=u_{z}^{(1)}(x, z)+u_{z}^{(2)}(x, z) \\
\phi(x, z)=\phi^{(1)}(x, z)+\phi^{(2)}(x, z)
\end{array}\right\}
$$


ここに，上付添字 (1) は式 (4) における $T(x, z)$ を $T^{(2)}(x, z)$ とした場合の特解, 上付添字 (2) は式 (4)に おいて温度項を零とした同次方程式の一般解を示寸。

熱弾性変位ポテンシャルを用いると, 特解が次のよ うに求まる。

$$
\begin{array}{r}
u_{x}^{(1)}(x, z)=\frac{m}{2 \pi} \int_{-\infty}^{\infty} \frac{L_{0}(s)}{s^{2}} \exp (-|s| \kappa z) \exp (-i s x) d s \\
+\frac{2 \kappa m}{\pi} \int_{0}^{\infty} \frac{1}{s^{2}}\left\{L_{1}(s) \exp (-s x / \kappa)\right. \\
\left.+2 L_{2}(s) \sinh (s x / \kappa)\right\} \sin (s z) d s \quad(14)
\end{array}
$$

$u_{z}^{(1)}(x, z)=-i \frac{\kappa k m}{2 \pi} \int_{-\infty}^{\infty} \frac{L_{0}(s)}{s|s|} \exp (-|s| \kappa z) \exp (-i s x) d s$

$$
-\frac{2 \kappa^{2} k m}{\pi} \int_{0}^{\infty} \frac{1}{s^{2}}\left\{L_{1}(s) \exp (-s x / \kappa)\right.
$$$$
\left.-2 L_{2}(s) \cosh (s x / \kappa)\right\} \cos (s z) d s
$$

$\phi^{(1)}(x, z)=i \frac{\kappa n}{2 \pi} \int_{-\infty}^{\infty} \frac{L_{0}(s)}{s|s|} \exp (-|s| \kappa z) \exp (-i s x) d s$

$$
\begin{aligned}
+\frac{2 \kappa^{2} n}{\pi} \int_{0}^{\infty} & \frac{1}{s^{2}}\left\{L_{1}(s) \exp (-s x / \kappa)\right. \\
& \left.-2 L_{2}(s) \cosh (s x / \kappa)\right\} \cos (s z) d s
\end{aligned}
$$

また, 一般解は Fourier 変換法により次のように求ま $ろ^{(19)}$.

$$
\begin{aligned}
u_{x}^{(2)}(x, z)= & \frac{i}{2 \pi} \sum_{j=1}^{3} \gamma_{j} a_{j} \int_{-\infty}^{\infty} \frac{|s|}{s} A_{j}(s) \exp \left(|s| \gamma_{j} z\right) \\
& \times \exp (-i s x) d s \\
- & \frac{2}{\pi} \sum_{j=1}^{3} \gamma_{j} a_{j} \int_{0}^{\infty}\left\{B_{1 j}(s) \exp \left(s x / \gamma_{j}\right)\right. \\
& \left.-2 B_{2 j}(s) \sinh \left(s x / \gamma_{j}\right)\right\} \sin (s z) d s \\
u_{z}^{(2)}(x, z)= & \frac{1}{2 \pi} \sum_{j=1}^{3} \int_{-\infty}^{\infty} A_{j}(s) \exp \left(|s| \gamma_{j} z\right) \\
& \times \exp (-i s x) d s \\
+\frac{2}{\pi} \sum_{j=1}^{3} \int_{0}^{\infty}\left\{B_{1 j}(s) \exp \left(s x / \gamma_{j}\right)\right. & \left.-2 B_{2 j}(s) \cosh \left(s x / \gamma_{j}\right)\right\} \cos (s z) d s \\
\phi^{(2)}(x, z)= & -\frac{1}{2 \pi} \sum_{j=1}^{3} b_{j} \int_{-\infty}^{\infty} A_{j}(s) \exp \left(|s| \gamma_{j} z\right) \\
& \times \exp (-i s x) d s \\
-\frac{2}{\pi} \sum_{j=1}^{3} b_{j} \int_{0}^{\infty}\left\{B_{1 j}(s) \exp \left(s x / \gamma_{j}\right)\right. & \left.-2 B_{2 j}(s) \cosh \left(s x / \gamma_{j}\right)\right\} \cos (s z) d s
\end{aligned}
$$

ここに, $A_{j}(s), B_{1 j}(s), B_{2 j}(s)(j=1,2,3)$ は境界条件式 より決定される未知関数, $m, k, n, \gamma_{j}, a_{j}, b_{j}(j=1,2,3)$ は文献 (15) の付録 1,2 に示卞既知定数である.

問題の解を特異積分方程式の解に導くため, 次の新 しい未知関数 $G(x)$ を導入する ${ }^{(20)}$.

$$
G(x)=\left\{\begin{array}{cr}
\frac{\partial}{\partial x} u_{x}^{(2)}(x, 0) & (a<x<b) \\
0 & (0 \leq x \leq a, b \leq x \leq h)
\end{array}\right\}
$$

境界条件式 (8), (9) を考慮すると, 混合境界条件式 (7) の第 1 式より, 未知関数 $G(t)$ に関する次の特異積分方 程式が得られる.

$\frac{1}{\pi} \int_{a}^{b} G(t)\left\{\frac{1}{t-x}-M_{1}(t, x)+M_{2}(t, x)\right\} d t=\frac{1}{\Gamma} \sigma_{z x}^{T}(x)$

$$
(a<x<b)
$$

式中の定数 $\Gamma$ および積分核 $M_{l}(t, x)(l=1,2)$ は次式 となる。

$$
\left.\begin{array}{l}
\Gamma=\sum_{j=1}^{3} p_{3 j} \delta_{j} \\
M_{1}(t, x)=\frac{1}{\delta_{B 0} \Gamma} \sum_{j=1}^{3} \sum_{k=1}^{3} p_{3 j} \delta_{B j k} \delta_{k} \frac{\gamma_{k}}{\gamma_{j} t+\gamma_{k} x} \\
M_{2}(t, x)=-\frac{2}{\Gamma} \sum_{j=1}^{3} \frac{p_{3 j}}{\gamma_{j}} \int_{0}^{\infty} \Gamma_{j}(s) \sinh \left(s x / \gamma_{j}\right) d s
\end{array}\right\}
$$

ここに, 定数 $p_{3 j}, \delta_{j}, \delta_{B 0}, \delta_{B j k}(j, k=1,2,3)$ および 関数 $\Gamma_{j}(s)(j=1,2,3)$ は付録 1 に示す. また, $\sigma_{z x}^{T}(x)$ は次のように得られる。

$$
\sigma_{z x}^{T}(x)=\sigma_{z x}^{T \infty}(x)+\sigma_{z x}^{T h}(x)
$$

上式中, 関数 $\sigma_{z x}^{T \infty}(x), \sigma_{z x}^{T h}(x)$ は付録 2 に示す.また, 混合境界条件式 (7) の第 2 式より, 次の補足の条件式 が得られる。

$$
\int_{a}^{b} G(t) d t=0
$$

特異積分方程式 (21) および補足の条件式 (24) を Gauss-Jacobi の数值積分公式 (21) を用いて数值解析 するために, 解の特異性を考慮し, $G(t)$ を滑らかな関 数 $\Phi(t)$ を用いて次のように置く.

$$
G(t)=\frac{c \Phi(t)}{\{(c+t)(c-t)\}^{1 / 2}}
$$

従って, 忘力拡大係数 $K_{I I a}, K_{I I b}$ は次のように求めら れる。

$$
\begin{aligned}
K_{I I a} & =\lim _{x \rightarrow a^{-}}\{2 \pi(x-a)\}^{1 / 2} \sigma_{z x}^{(2)}(x, 0) \\
& =-(\pi c)^{1 / 2} \Phi(a) \\
K_{I I b} & =\lim _{x \rightarrow b^{+}}\{2 \pi(b-x)\}^{1 / 2} \sigma_{z x}^{(2)}(x, 0) \\
& =(\pi c)^{1 / 2} \Phi(b)
\end{aligned}
$$




\section{4. 数値解析および考察}

数值計算を行い, 電気熱弾性場に及ぼすき裂位置 $(a+b) / 2 h$ およびき裂長さ $c / h$ の影響を解明寸る. 数 值計算例には, 次のセレン化カドミウムの電気熱弾性 特性(22)を用いた。

$$
\begin{aligned}
& c_{11}=7.41 \times 10^{10}[\mathrm{~Pa}], c_{33}=8.36 \times 10^{10}[\mathrm{~Pa}] \\
& c_{44}=1.32 \times 10^{10}[\mathrm{~Pa}] \\
& c_{12}=4.52 \times 10^{10}[\mathrm{~Pa}], c_{13}=c_{23}=3.93 \times 10^{10}[\mathrm{~Pa}] \\
& e_{31}=-0.16\left[\mathrm{C} / \mathrm{m}^{2}\right], e_{33}=0.347\left[\mathrm{C} / \mathrm{m}^{2}\right] \\
& e_{15}=-0.138\left[\mathrm{C} / \mathrm{m}^{2}\right] \\
& \varepsilon_{11}=0.825 \times 10^{-10}[\mathrm{C} / \mathrm{Vm}] \\
& \varepsilon_{33}=0.903 \times 10^{-10}[\mathrm{C} / \mathrm{Vm}] \\
& \alpha_{x}=\alpha_{y}=4.396 \times 10^{-6}\left[\mathrm{~K}^{-1}\right] \\
& \alpha_{z}=2.458 \times 10^{-6}\left[\mathrm{~K}^{-1}\right] \\
& p_{z}=-2.94 \times 10^{-6}\left[\mathrm{CK}^{-1} \mathrm{~m}^{-2}\right]
\end{aligned}
$$

ここに, $\alpha_{x}, \alpha_{y}, \alpha_{z}$ は線膨張係数であり, 温度応力係 数 $\lambda_{11}, \lambda_{33}$ は次式のように与えられる.

$$
\left.\begin{array}{l}
\lambda_{11}=c_{11} \alpha_{x}+c_{12} \alpha_{y}+c_{13} \alpha_{z} \\
\lambda_{33}=c_{13} \alpha_{x}+c_{23} \alpha_{y}+c_{33} \alpha_{z}
\end{array}\right\}
$$

また, 熱伝導率は, $\kappa^{2}=\kappa_{x} / \kappa_{z}=1 / 1.5$ と仮定した.

図 2 は, き裂面 $z \rightarrow 0^{+}$およびき裂延長線上にお ける基隻温度 $T_{0}=q_{0} c / \kappa_{z}$ で標準化された温度分布 $T(x, z) / T_{0}$ に及ぼす $c / h$ の影響を示したグラフであ り, $(a+b) / 2 h=0.3$ とした. $T(x, z) / T_{0}$ の最大值は, $c / h$ の增大により増加する。

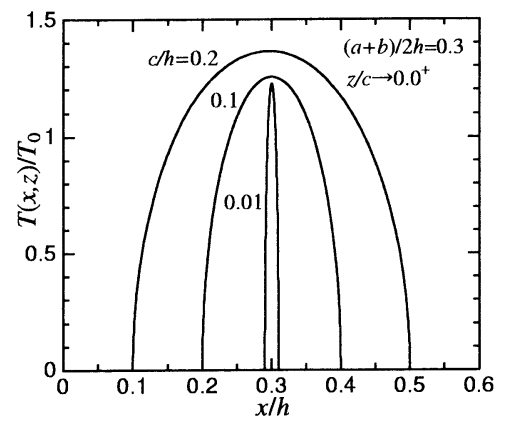

Fig.2 The temperatures on the crack face and the crack extended line for $(a+b) / 2 h=0.3$.

図 3,4 は, それぞれ特異積分方程式 (21) の右辺に ある $x$ 軸上の熱応力成分を標準化した $\sigma_{z x}^{T}(x) / T_{0} \lambda_{33}$ に及ぼす $c / h((a+b) / 2 h=0.3)$ および $(a+b) / 2 h$ $(c / h=0.1)$ の影響を示したグラフである. 実線およ び破線は, それぞれ本報の結果, 厚板中の単一き裂の 結果 (16) を示す.いずれも, 最大值および最小值は, き 裂先端 $(x=a, b)$ で発生している. また, $c / h$ の増大
あるいは $(a+b) / 2 h$ の減少により，き裂間相互干渉の 影響が䡛著になると, 単一き裂の結果に比べ, 応力分 布は正の向きに移動することが分かる。

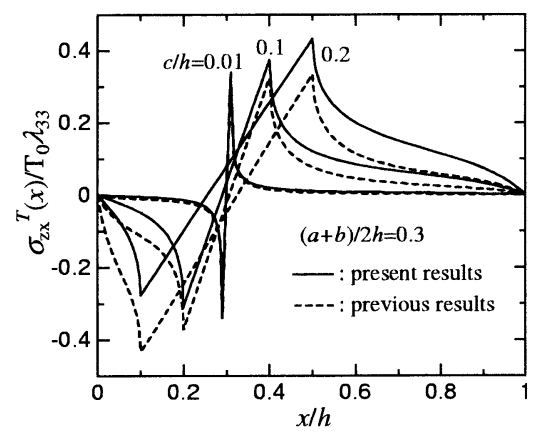

Fig. 3 The stress component on the $x$-axis without crack due to the temperatures shown in Figure 2.

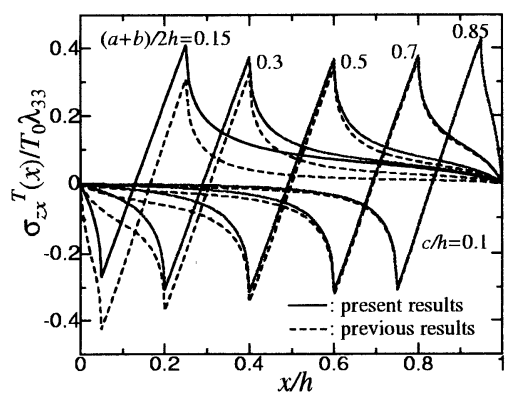

Fig.4 The stress component on the $x$-axis without crack for $c / h=0.1$.

図 5 は標準化された熱応力拡大係数 $\left(-K_{I I a}, K_{I I b}\right) /$ $\lambda_{33} T_{0}(\pi c)^{1 / 2}$ に及ぼす $c / h$ の影響を示したグラフで あり，実線および破線は，それぞれ $K_{I I a}, K_{I I b}$ を示す， $c / h \rightarrow 0.0$ では, $\left(-K_{I I a}, K_{I I b}\right) / \lambda_{33} T_{0}(\pi c)^{1 / 2} \rightarrow 0.171$ となる. $c / h$ の増大に伴って, $\left(-K_{I I a}, K_{I I b}\right) / \lambda_{33} T_{0}$ $(\pi c)^{1 / 2}$ は単調に増加または減少するが, $K_{I I b} / \lambda_{33} T_{0}(\pi c)^{1 / 2}$ に及ぼす $c / h$ の影響は $-K_{I I a} / \lambda_{33} T_{0}(\pi c)^{1 / 2}$ に比べて大である.

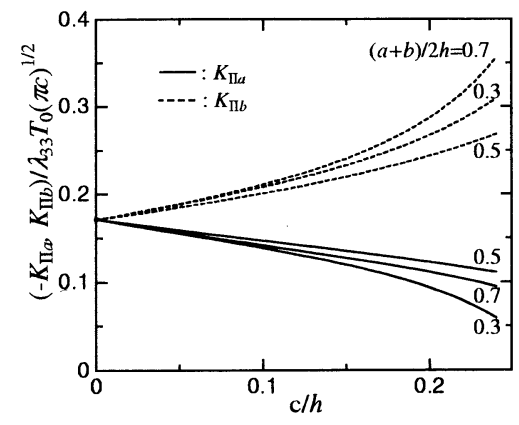

Fig.5 The effect of the crack length on the thermal stress intensity factors. 
図 6 は標準化された熱応力桩大係数 $\left(-K_{I I a}, K_{I b}\right) /$ $\lambda_{33} T_{0}(\pi c)^{1 / 2}$ に及ぼす $(a+b) / 2 h$ の影響を示したグラ フである. 実線および破線は，それぞれ本報の結果, 前 報 (単一き裂) $)^{(16)}$ の結果を示寸. $-K_{I I a}$ は $(a+b) / 2 h$ の増大に伴い, 増加した後, 最大值をとり, その後, 减 少するが, $K_{I I b}$ は $-K_{I I a}$ と逆の傾向をとっている.二 個のき裂が接近した場合，|KIIa | はき裂間相互干涉 のため減少し. 前報 (16) の結果とは大きく異なるが, き裂が自由表面近傍にある場合には，き裂閒相互干涉 の影響が減少するため, 前報(16) の結果に漸近する.

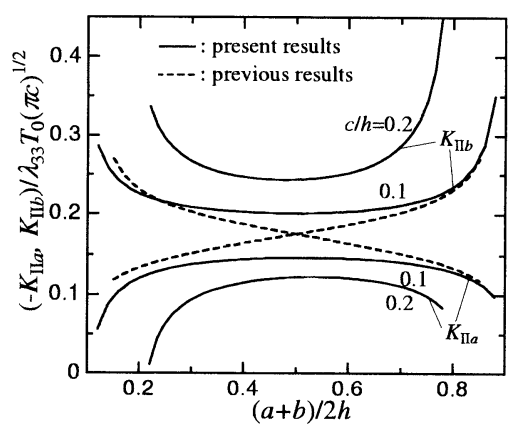

Fig.6 The effect of the crack location on the thermal stress intensity factors.

\section{5. 結}

本報は，二個の共線等長き裂が自由表面に対して垂 直に存在する圧電厚板に一様熱流が作用する場合の特 異熱応力問題を理論解析したものである. 数值計算を 行い, 温度場, 熱応力および熱応力拡大係数に及ぼす き裂位置およびき裂長さの影響を定量的に明らかにし た. 数値結果より, (1) 標準化された熱応力拡大係数は 幾何学的形状に大きな影響を受けること, (2) 二個のき 裂が接近した場合, $x=a$ での熱応力拡大係数の絶対 值は, き裂間相互干渉により減少すること, (3) 自由表 面近傍にあるき裂先端の熱応力拡大係数は, 前報 (16) で扱った単一き裂の結果と非常に近い値を示すことな どを明らかにした。

\section{付 録 1}

定数 $p_{k j}(j=1,2,3, k=1 \sim 5), \delta_{j}, \delta_{B 0}, \delta_{B j k}(j$, $k=1,2,3)$ は次式で与えられる.

$$
\left.\begin{array}{l}
p_{1 j}=c_{11} a_{j}+c_{13}-e_{31} b_{j} \\
p_{2 j}=c_{13} a_{j}+c_{33}-e_{33} b_{j} \\
p_{3 j}=c_{44}\left(\gamma_{j}^{2} a_{j}-1\right)+e_{15} b_{j} \\
p_{4 j}=e_{15}\left(\gamma_{j}^{2} a_{j}-1\right)-\varepsilon_{11} b_{j} \\
p_{5 j}=e_{31} a_{j}+e_{33}+\varepsilon_{33} b_{j}
\end{array}\right\}(j=1,2,3)
$$

$\delta_{1}=\rho_{22} \rho_{33} /\left(\rho_{22} \rho_{33} \gamma_{1} a_{1}-\rho_{21} \rho_{33} \gamma_{2} a_{2}-\rho_{22} \rho_{31} \gamma_{3} a_{3}\right)$ $\delta_{j}=-\rho_{j 1} \delta_{1} / \rho_{j, j}$

$\delta_{B 0}=\rho_{B 22} \rho_{B 33} \gamma_{1} a_{1}-\rho_{B 21} \rho_{B 33} \gamma_{2} a_{2}-\rho_{B 22} \rho_{B 31} \gamma_{3} a_{3}$ $\delta_{B 1 k}=-\left(\rho_{B 22} \rho_{B 33} \gamma_{k} a_{k}+\rho_{B 2 k} \rho_{B 33} \gamma_{2} a_{2}\right.$

$\delta_{B j k}=-\left(\rho_{B j 1} \delta_{B 1 k}-\rho_{B j k} \delta_{B 0}\right) / \rho_{B j}$ $\left.+\rho_{B 22} \rho_{B 3 k} \gamma_{3} a_{3}\right)$

$$
(j=2,3, k=1,2,3)
$$

ここに,

$$
\left.\begin{array}{l}
\rho_{2 j}=\gamma_{j}\left(p_{2 j} p_{53}-p_{23} p_{5 j}\right) \\
\rho_{3 j}=\gamma_{j}\left(p_{2 j} p_{52}-p_{22} p_{5 j}\right) \\
\rho_{B 1 j}=\left(p_{3 j} p_{41}-p_{31} p_{4 j}\right) / \gamma_{j} \\
\rho_{B 2 j}=\left(p_{3 j} p_{43}-p_{33} p_{4 j}\right) / \gamma_{j} \\
\rho_{B 3 j}=\left(p_{3 j} p_{42}-p_{32} p_{4 j}\right) / \gamma_{j}
\end{array}\right\}(j=1,2,3)
$$

関数 $\Gamma_{j}(s)(j=1,2,3)$ は次式で表す.

$$
\left.\begin{array}{rl}
\Gamma_{1}(s) & =\frac{Z_{1}(s)}{Z_{0}(s)} \\
\Gamma_{j}(s) & =\frac{f_{j}(s)}{2 \rho_{B j j} \sinh \left(s h / \gamma_{j}\right)} \quad(j=2,3)
\end{array}\right\}
$$

$$
こ こ に
$$

$$
\begin{aligned}
& Z_{0}(s)=-2\left\{p_{11} \cosh \left(s h / \gamma_{1}\right)\right. \\
& \left.-\sum_{j=2}^{3} p_{1 j} \frac{\rho_{B j 1}}{\rho_{B j j}} \frac{\sinh \left(s h / \gamma_{1}\right)}{\sinh \left(s h / \gamma_{j}\right)} \cosh \left(s h / \gamma_{j}\right)\right\} \\
& Z_{1}(s)=\frac{1}{\delta_{B 0}} \sum_{j=2}^{3} \sum_{k=1}^{3} \delta_{k} \frac{p_{1, j} \cosh \left(s h / \gamma_{j}\right)}{\rho_{B j j} \sinh \left(s h / \gamma_{j}\right)} \\
& \times\left[\left\{\rho_{B j 1} \delta_{B 1 k} \exp \left(s h / \gamma_{1}\right)\right.\right. \\
& \left.+\rho_{B j j} \delta_{B j k} \exp \left(s h / \gamma_{j}\right)\right\} F_{1 k}(s) \\
& \left.+\rho_{B j k} \delta_{B 0} F_{2 k}(s)\right] \\
& \left.\begin{array}{r}
-\frac{1}{\delta_{B 0}} \sum_{k=1}^{3} \delta_{k}\left\{\sum_{j=1}^{3} p_{1 j} \delta_{B j k} \exp \left(s h / \gamma_{j}\right) F_{1 k}(s)\right. \\
\left.+p_{1 k} \delta_{B 0} F_{2 k}(s)\right\}
\end{array}\right\}
\end{aligned}
$$

$$
\begin{aligned}
& f_{j}(s)=-2 \rho_{B j 1} \sinh \left(s h / \gamma_{1}\right) \Gamma_{1}(s) \\
& +\frac{1}{\delta_{B 0}} \sum_{k=1}^{3} \delta_{k}\left[\left\{\rho_{B j 1} \delta_{B 1 k} \exp \left(s h / \gamma_{1}\right)\right.\right. \\
& \left.\left.+\rho_{B j j} \delta_{B j k} \exp \left(s h / \gamma_{j}\right)\right\} F_{1 k}(s)+\rho_{B j k} \delta_{B 0} F_{2 k}(s)\right] \\
& (j=1,2,3)
\end{aligned}
$$

また，

$$
\left.\begin{array}{l}
F_{1 k}(s)=\exp \left(s t / \gamma_{k}\right) \\
\left.F_{2 k}(s)=\exp \left\{s(h-t) / \gamma_{k}\right)\right\}
\end{array}\right\} \quad(k=1,2,3)
$$




\section{付 録 2}

関数 $\sigma_{z x}^{T \infty}(x), \sigma_{z: x}^{T h}(x)$ は次式で与えられる.

$$
\begin{aligned}
& \sigma_{z x}^{T \infty}(x)=- \frac{1}{\pi}\left[\sum_{j=1}^{3} p_{3 j} \delta_{j}^{p} \ln \left\{\frac{t+x}{\gamma_{j}(t-x)}\right\}\right. \\
&\left.+q_{3} \ln \left\{\frac{t+x}{\kappa(t-x)}\right\}\right] \int_{a}^{b} G_{0}(t) d t \\
& \sigma_{z x}^{T h}(x)=\frac{2}{\pi} \int_{0}^{\infty}\left\{\sum_{j=1}^{3} \frac{p_{3 j}}{\gamma_{j}} \frac{C_{j}(s)}{s} \sinh \left(s x / \gamma_{j}\right)\right. \\
&\left.+2 q_{3} \frac{L_{2}(s)}{s} \sinh (s x / \kappa)\right\} \cos (s z) d s
\end{aligned}
$$

ここに,

$$
\left.\begin{array}{l}
q_{1}=m\left(c_{11}-\kappa^{2} k c_{13}\right)+\kappa^{2} n e_{31}-\lambda_{11} \\
q_{2}=m\left(c_{13}-\kappa^{2} k c_{33}\right)+\kappa^{2} n e_{33}-\lambda_{33} \\
q_{3}=\kappa m c_{44}(1+k)-\kappa n e_{15} \\
q_{4}=\kappa m e_{15}(1+k)+\kappa n \varepsilon_{11} \\
q_{5}=m\left(e_{31}-\kappa^{2} k e_{33}\right)-\kappa^{2} n \varepsilon_{33}+p_{z}
\end{array}\right\}
$$$$
\delta_{1}^{p}=\frac{\rho_{22} \rho_{33} m-\rho_{20} \rho_{33} \gamma_{2} a_{2}-\rho_{22} \rho_{30} \gamma_{3} a_{3}}{\rho_{22} \rho_{33} \gamma_{1} a_{1}-\rho_{21} \rho_{33} \gamma_{2} a_{2}-\rho_{22} \rho_{31} \gamma_{3} a_{3}}
$$$$
\delta_{j}^{p}=-\left(\rho_{j 1} \delta_{1}^{p}-\rho_{j 0}\right) / \rho_{j j}
$$

$$
(j=2,3)
$$

$$
\left.\begin{array}{l}
C_{1}(s)=\frac{Z_{1}^{T}(s)}{Z_{0}(s)} \\
C_{j}(s)=\frac{f_{j}^{T}(s)}{2 \rho_{B j j} \sinh \left(s h / \gamma_{j}\right)} \quad(j=2,3)
\end{array}\right\}
$$

また，

$$
\left.\begin{array}{c}
Z_{1}^{T}(s)=\sum_{j=2}^{3} \frac{p_{1 j} \cosh \left(s h / \gamma_{j}\right)}{\rho_{B j j} \sinh \left(s h / \gamma_{j}\right)}\left\{\rho_{B j 1} \delta_{B 11}^{p} \exp \left(s h / \gamma_{1}\right)\right. \\
\left.+\rho_{B j j} \delta_{B 1 j}^{p} \exp \left(s h / \gamma_{j}\right)+\rho_{B j 0}^{\prime}\right\} \\
+2 q_{1} \frac{1}{\sinh (s h / \kappa)} F_{3}^{\prime}(s) \\
-\sum_{j=1}^{3} p_{1 j}\left\{\gamma_{j} \delta_{j}^{p} F_{2 j}^{\prime}(s)+\delta_{B 1 j}^{p} \exp \left(s h / \gamma_{j}\right)\right\}+F_{0}^{\prime}
\end{array}\right\}
$$

$$
\begin{aligned}
& F_{0}^{\prime}=\left(\sum_{k=1}^{3} \gamma_{k} p_{1 k} \delta_{k}^{p}-q_{1}\right) \int_{a}^{b} G_{0}(t) d t \\
& F_{1 j}^{\prime}=\int_{a}^{b} G_{0}(t) \exp \left(s t / \gamma_{j}\right) d t \\
& F_{2 j}^{\prime}(s)=\int_{a}^{b} G_{0}(t) \exp \left\{s(h-t) / \gamma_{j}\right\} d t \\
& F_{3}^{\prime}(s)=\int_{a}^{b} G_{0}(t) \sinh (s t / \kappa) d t \\
& (j=1,2,3)
\end{aligned}
$$

ここに, $G_{0}(x)$ は前報 (16) の特異積分方程式 (14) の解 である。

\section{文献}

(1) Rao, S.S., Sunar, M., Piezoelectricity and Its Use in Disturbance Sensing and Control of Flexible Structures : A Survey, Applied Mechanics Review, Vol.47 (1994), pp.113-123.

(2) Wang, B.L., Mai, Y.W., A Cracked Piezoelectric Material Strip under Transient Thermal Loading, Transactions of the ASME, Journal of Applied Mechanics, Vol.69 (2002), pp.539-546.

(3) Niraula, O.P., Noda, N., Thermal Stress Analysis in Thermopiezoelastic Strip with an Edge Crack, Journal of Thermal Stresses, Vol.25 (2002), pp.389-405.

(4) Ueda, S., Kanada, Y., Piezothermoelastic Analysis of a Piezoelectric Laminated Slab with a Finite Crack, Transactions of the Japan Society of Mechanical Engineers, Series A, Vol.69, No.684 (2003), pp.1291-1297. (in Japanese)

(5) Ueda, S., Ikawa, K., The Crack Problem in Piezoelectric Strip under Thermoelectric Loading, Transactions of the Japan Society of Mechanical Engineers, Series A, Vol.72, No.715 (2006), pp.309-316. (in Japanese)

(6) Ueda, S., Ikawa, K., Transient Response of a Cracked Piezoelectric Strip under Thermoelectric Loading, Transactions of the Japan Society of Mechanical Engineers, Series A, Vol.72, No.720 (2006), pp.1169-1176. (in Japanese)

(7) Wang, B.L., Noda, N., The Exact Thermoelectroelasticity Solution for a Penny-Shaped Crack in Piezothermoelectric Materials, Journal of Thermal Stresses, Vol.27 (2004), pp.241-251.

(8) Wang, B.L., Noda, N., Thermally Induced Fracture of a Smart Functionally Graded Composite Structure, Theoretical and Applied Fracture Mechanics, Vol.35 (2001), pp.93-109.

(9) Ueda, S. et al., Thermo-electro-mechanical Interactions of a Functionally Graded Piezoelectric Plate with a Finite Crack, Transactions of the Japan Society of Mechanical Engineers, Series A, Vol.70, No.694 (2004), pp.872-879. (in Japanese)

(10) Ueda, S., Takaki, T., Thermoelectroelastic Response of a Symmetric Functionally Graded Piezoelectric Slab with a Crack Parallel to the Boundaries, Transactions of the Japan Society of Mechanical Engineers, Series A, Vol.72, No.720 (2006), pp.1177-1184. (in Japanese) 
(11) Ueda, S., Kondo, H., Thermoelectroelastic Response of a Functionally Graded Piezoelectric Strip Containing a Crack Parallel to the Free Boundaries, Transactions of the Japan Society of Mechanical Engineers, Series A, Vol.72, No.722 (2006), pp.1503-1510. (in Japanese)

(12) Ueda, S., Kondo, H., Thermal Shock Response a Parallel Crack in a Functionally Graded Piezoelectric Material Plate, Transactions of the Japan Society of Mechanical Engineers, Series A, Vol.73, No.727 (2007), pp.387-394. (in Japanese)

(13) Ueda, S., et al., Thermoelectromechanical Response of a Penny-Shaped Crack in a Functionally Graded Piezoelectric Strip, Transactions of the Japan Society of Mechanical Engineers, Series A, Vol.73, No.728 (2007), pp.506-513. (in Japanese)

(14) Ueda, S., et al., Transient Thermoelectroelastic Response of a Functionally Graded Piezoelectric Strip with a Penny-Shaped Crack, Transactions of the Japan Society of Mechanical Engineers, Series A, Vol.73, No.733 (2007), pp.1013-1020. (in Japanese)

(15) Ueda, S., et al., Thermal Stress Intensity Factors for a Normal Crack in a Semi-Infinite Piezoelectric Material, Transactions of the Japan Society of Mechanical Engineers, Series A, Vol.72, No.716 (2006), pp.513-519. (in Japanese)

(16) Ueda, S., Tani, Y., Thermal Stress Intensity Factors for a Normal Crack in a Piezoelectric Strip, Transactions of the Japan Society of Mechanical Engineers, Series A, Vol.72, No.722 (2006), pp.1487-1494. (in Japanese)
(17) Chen, Z.T., Worswick, M.J., Antiplane Mechanical and Inplane Electric Time-Dependent Load Applied to Two coplanar Cracks in Piezo-electric Ceramic Material, Theoretical and Applied Fracture Mechanics, Vol.33 (2000), pp.173-184.

(18) Wang, B.L., Mai, Y.W., Impermeable Crack and Permeable Crack Assumptions, Which One is More Realistric? Transactions of the ASME, Journal of Applied Mechanics, Vol.71 (2004), pp.575-578.

(19) Sneddon, I.N., Lowengrub, M., Crack Problems in the Classical Theory of Elasticity, (1969), John Wiley \& Sons, Inc., New York.

(20) Erdogan, F., Wu, B.H., Crack Problems in FGM Layers under Thermal Stresses, Journal of Thermal Stresses, Vol.19 (1996), pp.237-265.

(21) Erdogan, F. et al., Methods of Analysis and Solution of Crack Problems, (Edited by G.C.Sih), (1972), Noordhoff, Leyden.

(22) Ashida, F., Tauchert, T.R., Transient Response of a Piezothermoelastic Circular Disk under Axisymmetric Heating, Acta Mechanica., Vol.128 (1998), pp.1-14. 